\title{
On the $X^{1} \Sigma^{+}$rovibrational spectrum of lithium hydride
}

\author{
Y. B. Shi ${ }^{1,2}$, P. C. Stancil ${ }^{1}$, and J. G. Wang ${ }^{2}$ \\ 1 Department of Physics and Astronomy and the Center for Simulational Physics, University of Georgia, Athens, GA 30602, USA \\ e-mail: stancil@physast.uga.edu \\ 2 Institute of Applied Physics and Computational Mathematics, 100094 Beijing, PR China \\ e-mail: [shi_yanbo;wang_jianguo] @iapcm.ac.cn
}

Received 26 April 2012 / Accepted 21 January 2013

\begin{abstract}
The complete line list of rovibrational transitions of the $\mathrm{X}^{1} \Sigma^{+}$state of $\mathrm{LiH}$ is computed. The line list includes all possible dipoleallowed transition energies and oscillator strengths that cover the transition energy range $3.27-19476 \mathrm{~cm}^{-1}$. The line list was obtained using an accurate potential and dipole moment function constructed from available experimental and theoretical data. This paper discusses the agreement of the current calculations with previous theoretical and experimental results. We also provide the radiative cooling function in the high-density limit over a wide temperature range and compare them with previous results. A simulated collisionally-broadened $\mathrm{LiH}$ opacity relevant to cool dwarf stars is also presented.
\end{abstract}

Key words. molecular data - molecular processes

\section{Introduction}

Lithium hydride has been the subject of theoretical and experimental molecular physics investigations for many years (see for example Partridge \& Langhoff 1981). As the simplest neutral heteronuclear diatomic molecule, $\mathrm{LiH}$ is a favorite benchmark for various quantum-chemical techniques (Tung et al. 2011; Holka et al. 2011). In astrophysics, $\mathrm{LiH}$ may play a role at temperatures $T \leq 5000 \mathrm{~K}$ in the cooling of primordial clouds (Stancil et al. 1996; Bougleux \& Galli 1997; Galli \& Palla 1998; Bovino et al. 2011) and is a means of monitoring the evolution of stars and interstellar clouds (Dulick et al. 1998). Furthermore, $\mathrm{LiH}$, as well as other Li-bearing molecules ( $\mathrm{LiCl}$ and $\mathrm{LiOH})$, may be observable in cool $\left(T_{\text {eff }} \leq 2000 \mathrm{~K}\right)$ dwarf atmospheres (Lodders 1999; Weck et al. 2004). However, to date, searches for $\mathrm{LiH}$ at high redshift have proved unsuccessful (Friedel et al. 2011). Reviews of the lithium chemistry in the early Universe have been given by Lepp et al. (2002) and Bovino et al. (2011), while the status of laboratory infrared spectroscopy of $\mathrm{LiH}$ is outlined in Dulick et al. (1998). A complete and evaluated rovibrational line list for the ground electronic state, which is germane to its thermal evolution and needed for primordial spectral models, is needed ${ }^{1}$.

In Sect. 2 we present the details of potential energy curve and dipole moment function for the electronic ground state of $\mathrm{LiH}$. In Sect. 3, we briefly outline the relevant relations for computing transition energies, Einstein coefficients, oscillator strengths, and radiative cooling functions. Results of the current computation including the ground electronic-state rovibrational line list and cooling function are presented in Sect. 4 along with comparisons to previous work and astrophysical applications. Section 5 gives a summary of the current work. Atomic units are used throughout, unless otherwise noted.

\footnotetext{
1 A previous, though unevaluated, line list is available on the ExoMol database: http://www. exomol.com
}

\section{Potential energy and dipole moment function}

Within the well-known Born-Oppenheimer approximation, the motion of a diatomic molecule may be partitioned into its electronic and nuclear components. As such, the non-relativistic Hamiltonian of a molecule may be written as

$\hat{H}=\hat{H}_{\mathrm{e}}+\hat{T}_{\mathrm{N}}$

with the time-independent Schrödinger equation given by

$\hat{H} \Psi(\boldsymbol{r} ; \boldsymbol{R})=E \Psi(\boldsymbol{r} ; \boldsymbol{R})$.

By separation of variables, the total wave function may be written as

$\Psi(\boldsymbol{r} ; \boldsymbol{R})=\Psi_{\mathrm{e}}(\boldsymbol{r} ; \boldsymbol{R}) \Psi_{\mathrm{N}}(\boldsymbol{R})$,

where $\boldsymbol{R}$ is the internuclear distance vector in body-fixed coordinates, and $\boldsymbol{r}$ the electron coordinate vector with respect to the center of mass of the nuclei. The motion of electrons (e) and nuclei $(\mathrm{N})$ can be described by the electronic Schrödinger equation

$\hat{H}_{\mathrm{e}} \Psi_{\mathrm{e}}(\boldsymbol{r} ; \boldsymbol{R})=E_{\mathrm{e}}(R) \Psi_{\mathrm{e}}(\boldsymbol{r} ; \boldsymbol{R})$

and the nuclear Schrödinger equation

$\left[\hat{T}_{\mathrm{N}}+E_{\mathrm{e}}(\boldsymbol{R})\right] \Psi_{\mathrm{N}}(\boldsymbol{R})=E_{v J} \Psi_{\mathrm{N}}(\boldsymbol{R})$,

where $E_{\mathrm{e}}(\boldsymbol{R})$ is the potential energy and $E_{v, J}$ the rovibrational energy or eigenvalue.

The dipole moment function can be defined as

$\boldsymbol{D}(\boldsymbol{R})=\int \Psi_{\mathrm{e}}^{*}(\boldsymbol{r} ; \boldsymbol{R})\left(\sum_{i} e \boldsymbol{r}_{i}\right) \Psi_{\mathrm{e}}(\boldsymbol{r} ; \boldsymbol{R}) \mathrm{d} r$,

where $\Psi_{\mathrm{e}}(\boldsymbol{r} ; \boldsymbol{R})$ is the electronic eigenfunction. In this work we consider $X^{1} \Sigma^{+} \leftarrow X^{1} \Sigma^{+}$rovibrational transitions so that only the $z$-component of the $\boldsymbol{r}$ operator contributes. 


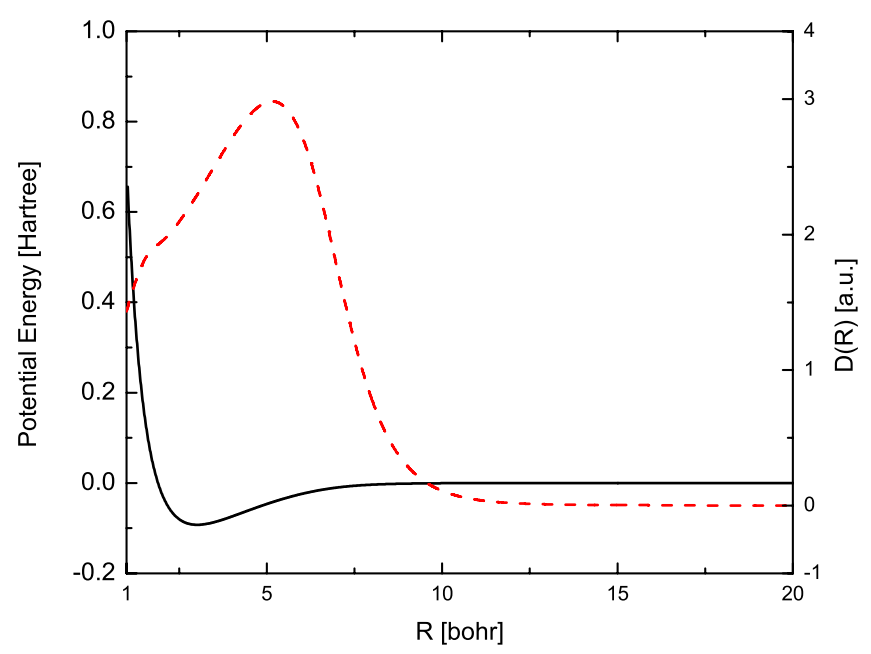

Fig. 1. Potential energy (solid line) and the dipole moment function (dashed line) of the $\mathrm{X}^{1} \Sigma^{+}$state for $\mathrm{LiH}$.

For a diatomic molecule, the potential energy is only a function of nuclear distance and can be written as $E_{\mathrm{e}}(R)$. We applied an empirical potential (Coxon \& Dickinson 2004), which was generated by directly fitting the spectroscopic $\mathrm{LiH}$ line positions, for $2<R<20 a_{0}$. The long-range potential is given by $V^{\mathrm{LR}}(R)=-\frac{C_{6}}{R^{6}}-\frac{C_{8}}{R^{8}}-\frac{C_{10}}{R^{10}}$ for $R>20 a_{0}$, where $C_{6}=66.536$, $C_{8}=3279.99$ and $C_{10}=223016.6$ (Yan et al. 1996). For $R<2 a_{0}$, the potential has been extrapolated with the shortrange form $V^{\mathrm{SR}}(R)=A \exp (-B R)+C$.

In a similar way, the dipole moment function of the $X$ state calculated by Partridge \& Langhoff (1981) has been used over the range $R=1.75$ to $17.5 a_{0}$. For $R>17.5 a_{0}$, the longrange form of Bottcher \& Dalgarno (1974), $D^{\mathrm{LR}}(R)=d_{7} / R^{7}$, was adopted. For $R<1.75 a_{0}$, the short-range form $D^{\mathrm{SR}}(R)=$ $A R^{2}+B R$ was fit to the ab initio data. The potential and dipole moment function are displayed in Fig. 1.

\section{Rovibrational eigenvalues, transition probabilities, and related quantities}

The radial nuclear Schrödinger equation of a diatomic molecule may be written as

$\left[-\frac{1}{2 \mu} \frac{\mathrm{d}^{2}}{\mathrm{~d} R^{2}}+E_{\mathrm{e}}(R)+\frac{J(J+1)}{2 \mu R^{2}}-E_{v J}\right] \chi_{v J}(R)=0$,

which can be solved numerically by the Numerov-Cooley method (Johnson 1977), for example. Here $\mu$ is the reduced mass of ${ }^{7} \mathrm{LiH}, v$ is vibrational quantum number, $J$ the rotational quantum number, and $\chi_{v J}(R)$ is the corresponding rovibrational eigenfunction. The Einstein A coefficient, or transition probability, in the dipole approximation, can be calculated given $\chi_{v J}(R)$ and $D(R)$, and is defined as (Weissbluth 1978)

$A_{v^{\prime} J^{\prime}, v^{\prime \prime} J^{\prime \prime}}=\frac{4}{3} \frac{c \alpha^{4}}{a_{0}} \Delta E_{v^{\prime} J^{\prime}, v^{\prime \prime} J^{\prime \prime}}^{3} \frac{S_{J^{\prime} J^{\prime \prime}}}{2 J^{\prime \prime}+1}$

where $\alpha=e^{2} / \hbar c$, is the fine-structure constant, $a_{0}$ is the Bohr radius, and $\Delta E_{v^{\prime} J^{\prime}, v^{\prime \prime} J^{\prime \prime}}$ the transition energy. Here $S_{J^{\prime} J^{\prime \prime}}$ is the line strength and defined as

$S_{J^{\prime} J^{\prime \prime}}=\left|\left\langle\chi_{v^{\prime} J^{\prime}}|D(R)| \chi_{v^{\prime \prime} J^{\prime \prime}}\right\rangle\right|^{2} \varphi_{J^{\prime} J^{\prime \prime}}$.
Table 1. Vibrational binding energies $G_{v^{\prime}}$ of the $X^{1} \Sigma^{+}$state of ${ }^{7} \mathrm{LiH}$ in units of $\mathrm{cm}^{-1}$.

\begin{tabular}{ccccc}
\hline \hline$v$ & $J_{\max }{ }^{a}$ & Present & Experiment $^{b}$ & Holka $^{c}$ \\
\hline 0 & 61 & 19588.05 & 19589.820 & 19593.55 \\
1 & 59 & 18228.34 & 18230.110 & 18232.97 \\
2 & 57 & 16913.49 & 16915.220 & 16917.44 \\
3 & 55 & 15642.58 & 15644.330 & 15646.02 \\
4 & 54 & 14414.77 & 14416.560 & 14417.86 \\
5 & 52 & 13229.32 & 13231.120 & 13232.2 \\
6 & 50 & 12085.58 & 12087.350 & 12088.36 \\
7 & 48 & 10983.01 & 10984.750 & 10985.82 \\
8 & 46 & 9921.24 & 9922.970 & 9924.17 \\
9 & 44 & 8900.06 & 8901.800 & 8903.2 \\
10 & 42 & 7919.47 & 7921.280 & 7922.91 \\
11 & 40 & 6979.78 & 6981.660 & 6983.55 \\
12 & 38 & 6081.62 & 6083.570 & 6085.76 \\
13 & 36 & 5226.11 & 5228.090 & 5230.59 \\
14 & 33 & 4414.92 & 4416.900 & 4419.72 \\
15 & 31 & 3650.52 & 3652.460 & 3655.55 \\
16 & 28 & 2936.31 & 2938.340 & 2941.52 \\
17 & 25 & 2277.00 & 2278.970 & 2282.34 \\
18 & 23 & 1678.99 & 1681.080 & 1684.42 \\
19 & 20 & 1151.01 & 1153.170 & 1156.35 \\
20 & 16 & 704.78 & 706.560 & 709.36 \\
21 & 12 & 354.95 & 355.570 & 357.48 \\
22 & 8 & 115.39 & 117.860 & 117.34 \\
\hline
\end{tabular}

Notes. ${ }^{(a)} J_{\max }$ is the maximum rotational level determined in the present calculation.

References. ${ }^{(b)}$ Chan et al. (1986). ${ }^{(c)}$ Holka et al. (2011).

Here $\varphi_{J^{\prime} J^{\prime \prime}}$ is a Hönl-London factor (Herzberg 1989) given by

$\varphi_{J^{\prime} J^{\prime \prime}}= \begin{cases}J^{\prime \prime}, & \text { P-branch } \\ J^{\prime \prime}+1, & \text { R-branch. }\end{cases}$

The oscillator strength may then be defined (Weissbluth 1978) as

$f_{v^{\prime} J^{\prime}, v^{\prime \prime} J^{\prime \prime}}=\frac{2}{3} \Delta E_{v^{\prime} J^{\prime}, v^{\prime \prime} J^{\prime \prime}} \frac{S_{J^{\prime} J^{\prime \prime}}}{2 J^{\prime \prime}+1}$.

In a variety of applications, it is useful to obtain the radiative cooling function in the high-density or local thermodynamic equilibrium (LTE) limit (see for example Coppola et al. 2011). In LTE, the radiative cooling function only depends on the rovibrational energies, degeneracies, and transition probabilities. It is only a function of the gas kinetic temperature $T$ and is given (in ergs/s) by

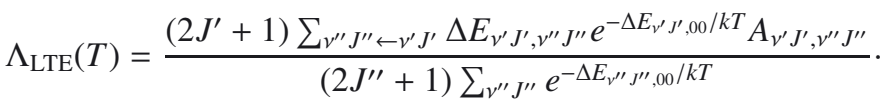

The sum is over all possible rovibrational deexcitation transitions within the electronic ground state and the denominator is the rovibrational partition function $Q(T)$.

\section{Results and discussion}

The radial Schrödinger Eq. (7) was solved with a step size $1.0 \times$ $10^{-3} a_{0}$, over a range of internuclear distances from $R=1.0$ to $60.0 a_{0}$. The reduced mass of ${ }^{7} \mathrm{LiH}$ was $0.881238162 \mathrm{u}$ (Stwalley \& Zemke 1993).

The vibrational energies $G_{\gamma^{\prime}}$ and corresponding vibrational energy spacings $\Delta G_{v^{\prime}+1 / 2}$ of the $X^{1} \Sigma^{+}$state obtained in the 
Y. B. Shi et al.: On the $\mathrm{X}^{1} \Sigma^{+}$rovibrational spectrum of lithium hydride

Table 2. Vibrational level spacings $\Delta G_{v^{\prime}+1 / 2}$ of the $\mathrm{X}^{1} \Sigma^{+}$state of ${ }^{7} \mathrm{LiH}$ where $\Delta G_{v^{\prime}+1 / 2}=G_{v^{\prime}+1}-G_{v^{\prime}}$ is in units of $\mathrm{cm}^{-1}$.

\begin{tabular}{lccc}
\hline \hline$v$ & Present & Experiment $^{a}$ & Holka $^{b}$ \\
\hline 0 & 1359.71 & 1359.71 & 1360.58 \\
1 & 1314.85 & 1314.89 & 1315.53 \\
2 & 1270.91 & 1270.89 & 1271.42 \\
3 & 1227.81 & 1227.77 & 1228.16 \\
4 & 1185.45 & 1185.44 & 1185.66 \\
5 & 1143.75 & 1143.77 & 1143.84 \\
6 & 1102.57 & 1102.60 & 1102.54 \\
7 & 1061.77 & 1061.78 & 1061.65 \\
8 & 1021.18 & 1021.17 & 1020.97 \\
9 & 980.58 & 980.52 & 980.29 \\
10 & 939.69 & 939.62 & 939.36 \\
11 & 898.16 & 898.09 & 897.79 \\
12 & 855.52 & 855.48 & 855.17 \\
13 & 811.18 & 811.19 & 810.87 \\
14 & 764.40 & 764.44 & 764.17 \\
15 & 714.21 & 714.12 & 714.03 \\
16 & 659.31 & 659.37 & 659.18 \\
17 & 598.01 & 597.89 & 597.92 \\
18 & 527.98 & 527.91 & 528.07 \\
19 & 446.23 & 446.61 & 446.99 \\
20 & 349.83 & 350.99 & 351.88 \\
21 & 239.56 & 237.71 & 240.14 \\
\hline
\end{tabular}

References. ${ }^{(a)}$ Chan et al. (1986). ${ }^{(b)}$ Holka et al. (2011).

present study are listed in Tables 1 and 2, along with the theoretical values of Holka et al. (2011) and the measurements of Chan et al. (1986). The largest discrepancy for $G_{v^{\prime}}$ between our calculations and the experiment is $2.47 \mathrm{~cm}^{-1}$, which occurs for $v=22$, while the largest difference for $\Delta G_{v^{\prime}+1 / 2}$ is smaller at $1.85 \mathrm{~cm}^{-1}$ for $v=22$. Some select transition energies are listed in Table 3 along with the corresponding experimental results of Dulick et al. (1998) and the calculations of Coppola et al. (2011). It can be seen that the current results are in excellent agreement with experiments, the largest discrepancy being $0.027 \mathrm{~cm}^{-1}$.

Transition probabilities for some band-averaged transitions in the $X^{1} \Sigma^{+}$state are compared in Table 4 with the previous calculation of Partridge \& Langhoff (1981). The maximum difference is less than $6.79 \%$. As a further illustration, the $J$-dependent dipole moment matrix elements for $v=1$ to $v=0$ are shown in Fig. 2. which are similar to those given in Gianturco et al. (1996). It can be seen that the transition probabilities for the P-branch are much higher than those for the R-branch. The line oscillator strengths as a function of wavelength are shown in Fig. 3 for the $\mathrm{X}^{1} \Sigma^{+} \leftarrow \mathrm{X}^{1} \Sigma^{+}$transition. The most intense line of the $\mathrm{R}$-branch is at $22.968 \mu \mathrm{m}$ (band 0-0, R(60)). For the P-branch, the strongest is at $16.822 \mu \mathrm{m}$ (band 8-7, $\mathrm{P}(31)$ ). The complete line list is available on the UGA Molecular Opacity Project website (http://www.physast.uga.edu/ugamop/) and in the format of the Leiden Atomic and Molecular Database (LAMDA, Schöier et al. 2005).

Utilizing the theoretical rovibrational energies and transition probabilities, the LTE radiative cooling function was calculated as a function of temperature as shown in Fig. 4. It is compared with the previous results of Dalgarno (1994, priv. comm.) and Coppola et al. (2011), along with the low-density limit cooling function $\left(n_{\mathrm{H}} \leq 100 \mathrm{~cm}^{-3}\right)$ due to $\mathrm{H}$ collisions (Galli \& Palla 1998) (see the Appendix for a further discussion). All three LTE cooling functions are seen to be in excellent agreement over the considered temperature range.
Table 3. Rovibrational transition energies for in the $X^{1} \Sigma^{+}$state of ${ }^{7} \mathrm{LiH}$.

\begin{tabular}{|c|c|c|c|c|c|}
\hline \multirow[b]{2}{*}{ Band } & \multirow[b]{2}{*}{ Line } & \multicolumn{2}{|c|}{ Transition energy $\left(\mathrm{cm}^{-1}\right)$} & \multirow[b]{2}{*}{$\Delta E_{\mathrm{cp}}{ }^{b}$} & \multirow[b]{2}{*}{$\Delta E^{c}$} \\
\hline & & Present & Experiment $^{a}$ & & \\
\hline \multirow[t]{11}{*}{$1-0$} & $\mathrm{P}(28)$ & 858.1193 & 858.0924 & 0.31 & $2.7 \mathrm{E}-02$ \\
\hline & $\mathrm{P}(23)$ & 953.1177 & 953.0989 & 0.38 & $1.9 \mathrm{E}-02$ \\
\hline & $\mathrm{P}(18)$ & 1048.2419 & 1048.231 & 0.46 & $1.1 \mathrm{E}-02$ \\
\hline & $\mathrm{P}(13)$ & 1141.6388 & 1141.6337 & 0.54 & $5.1 \mathrm{E}-03$ \\
\hline & $\mathrm{P}(8)$ & 1231.0873 & 1231.0857 & 0.63 & $1.6 \mathrm{E}-03$ \\
\hline & $\mathrm{P}(3)$ & 1314.0901 & 1314.0902 & 0.72 & $-1.0 \mathrm{E}-04$ \\
\hline & $\mathrm{R}(2)$ & 1401.5064 & 1401.5062 & 0.82 & $2.0 \mathrm{E}-04$ \\
\hline & $\mathrm{R}(7)$ & 1461.2703 & 1461.2695 & 0.88 & 8.0E-04 \\
\hline & $\mathrm{R}(12)$ & 1506.8725 & 1506.8724 & 0.93 & $1.0 \mathrm{E}-04$ \\
\hline & $\mathrm{R}(17)$ & 1536.7646 & 1536.7673 & 0.95 & $-2.7 \mathrm{E}-03$ \\
\hline & $R(22)$ & 1550.0615 & 1550.0721 & 0.94 & $-1.1 \mathrm{E}-02$ \\
\hline \multirow[t]{10}{*}{$2-1$} & $\mathrm{P}(27)$ & 844.9443 & 844.9214 & 0.33 & $2.3 \mathrm{E}-02$ \\
\hline & $\mathrm{P}(22)$ & 937.6974 & 937.6828 & 0.38 & $1.5 \mathrm{E}-02$ \\
\hline & $\mathrm{P}(17)$ & 1030.1894 & 1030.1815 & 0.45 & $7.9 \mathrm{E}-03$ \\
\hline & $\mathrm{P}(12)$ & 1120.5469 & 1120.5438 & 0.53 & $3.1 \mathrm{E}-03$ \\
\hline & $\mathrm{P}(7)$ & 1206.5402 & 1206.5394 & 0.62 & $8.0 \mathrm{E}-04$ \\
\hline & $\mathrm{P}(2)$ & 1285.6869 & 1285.6872 & 0.71 & $-3.0 \mathrm{E}-04$ \\
\hline & $\mathrm{R}(3)$ & 1368.0210 & 1368.0208 & 0.80 & $2.0 \mathrm{E}-04$ \\
\hline & $\mathrm{R}(8)$ & 1423.2838 & 1423.2834 & 0.86 & $4.0 \mathrm{E}-04$ \\
\hline & $\mathrm{R}(13)$ & 1464.2863 & 1464.2873 & 0.90 & $-1.0 \mathrm{E}-03$ \\
\hline & $\mathrm{R}(17)$ & 1485.8610 & 1485.8639 & 0.92 & $-2.9 \mathrm{E}-03$ \\
\hline \multirow[t]{9}{*}{$3-2$} & $\mathrm{p}(23)$ & 885.7266 & 885.7132 & 0.39 & $1.3 \mathrm{E}-02$ \\
\hline & $\mathrm{P}(18)$ & 976.0542 & 976.0472 & 0.44 & 7.0E-03 \\
\hline & $\mathrm{P}(12)$ & 1081.9646 & 1081.9626 & 0.53 & $2.0 \mathrm{E}-03$ \\
\hline & $\mathrm{P}(7)$ & 1165.6453 & 1165.645 & 0.61 & $3.0 \mathrm{E}-04$ \\
\hline & $\mathrm{P}(2)$ & 1242.5862 & 1242.5865 & 0.69 & $-3.0 \mathrm{E}-04$ \\
\hline & $\mathrm{R}(3)$ & 1322.4840 & 1322.4835 & 0.78 & 5.0E-04 \\
\hline & $\mathrm{R}(8)$ & 1375.9434 & 1375.9434 & 0.85 & $0.0 \mathrm{E}+00$ \\
\hline & $\mathrm{R}(13)$ & 1415.3862 & 1415.3881 & 0.89 & $-1.9 \mathrm{E}-03$ \\
\hline & $\mathrm{R}(18)$ & 1439.4378 & 1439.4452 & 0.92 & $-7.4 \mathrm{E}-03$ \\
\hline \multirow[t]{7}{*}{$4-3$} & $\mathrm{P}(21)$ & 888.1921 & 888.1827 & 0.42 & $9.4 \mathrm{E}-03$ \\
\hline & $\mathrm{P}(16)$ & 975.7737 & 975.7707 & 0.48 & $3.0 \mathrm{E}-03$ \\
\hline & $\mathrm{P}(11)$ & 1060.7999 & 1060.7993 & 0.55 & $6.0 \mathrm{E}-04$ \\
\hline & $\mathrm{P}(6)$ & 1141.0946 & 1141.0945 & 0.63 & $1.0 \mathrm{E}-04$ \\
\hline & $\mathrm{R}(1)$ & 1253.6893 & 1253.6882 & 0.75 & $1.1 \mathrm{E}-03$ \\
\hline & $R(6)$ & 1310.3681 & 1310.3676 & 0.82 & $5.0 \mathrm{E}-04$ \\
\hline & $\mathrm{R}(11)$ & 1353.9579 & 1353.9574 & 0.88 & $5.0 \mathrm{E}-04$ \\
\hline \multirow[t]{6}{*}{$5-4$} & $\mathrm{P}(15)$ & 957.1386 & 957.1371 & 0.51 & $1.5 \mathrm{E}-03$ \\
\hline & $\mathrm{P}(10)$ & 1039.1932 & 1039.1925 & 0.57 & $7.0 \mathrm{E}-04$ \\
\hline & $\mathrm{P}(4)$ & 1130.6447 & 1130.6442 & 0.66 & $5.0 \mathrm{E}-04$ \\
\hline & $\mathrm{R}(4)$ & 1244.8800 & 1244.8819 & 0.79 & $-1.9 \mathrm{E}-03$ \\
\hline & $\mathrm{R}(5)$ & 1255.3743 & 1255.3722 & 0.81 & $2.1 \mathrm{E}-03$ \\
\hline & $\mathrm{R}(8)$ & 1283.7835 & 1283.7859 & 0.85 & $-2.4 \mathrm{E}-03$ \\
\hline
\end{tabular}

Notes. ${ }^{(a)}$ Dulick et al. (1998). ${ }^{(b)}$ Comparison between Coppola et al. (2011) and Dulick et al. (1998), $\Delta E_{\mathrm{cp}}=E_{\mathrm{cp}}-E_{\text {expt }} \cdot{ }^{(c)} \Delta E=E_{\mathrm{th}}-E_{\text {expt }}$.

To simulate the opacity of $\mathrm{LiH}$ in a cool dwarf atmosphere, we computed the absorption cross section using Eq. (6) of Dulick et al. (2003) for a temperature of $1800 \mathrm{~K}$, as an example. The line absorption cross section, which is a function of the transition probability, line frequency, partition function, and temperature, is multiplied by a Lorentzian line profile (Bernath 1995) with width corresponding to collisional broadening. Assuming a canonical collisional broadening cross section of $10^{-16} \mathrm{~cm}^{2}$ with a line width proportional to the pressure, Figure 5 displays the opacity for 100 atms. There is a series of strong individual pure rotational lines for $\lambda>20 \mu \mathrm{m}$, while the fundamental vibrational band occurs near $8 \mu \mathrm{m}$. Comparison is made to the $\mathrm{LiCl}$ opacity (Weck et al. 2004) for the same temperature and pressure. The $\mathrm{LiCl}$ fundamental vibrational band falls in the gap between the pure $\mathrm{LiH}$ rotational lines and the $\mathrm{LiH} 0-1$ band, but is typically 
Table 4. A-values for transitions in the $X^{1} \Sigma^{+}$state of ${ }^{7} \mathrm{LiH}$.

\begin{tabular}{lcccc}
\hline \hline & \multicolumn{2}{c}{$A_{v^{\prime} \nu^{\prime \prime}}\left(\mathrm{s}^{-1}\right)$} & & \\
\cline { 2 - 4 }$v^{\prime}-v^{\prime \prime}$ & Present $^{a}$ & Coppola $^{b}$ & Partridge $^{c}$ & $\Delta(\%)^{d}$ \\
\hline $1-0$ & 45.4 & 46.6 & 43.1 & 5.32 \\
$2-0$ & 2.0 & 2.1 & 1.9 & 4.66 \\
$2-1$ & 82.8 & 85.3 & 78.5 & 5.43 \\
$3-1$ & 6.4 & 6.6 & 6.3 & 2.15 \\
$3-2$ & 112.5 & 115.9 & 106.7 & 5.47 \\
$4-2$ & 13.4 & 13.6 & 13.3 & 0.72 \\
$4-3$ & 134.5 & 138.7 & 127.3 & 5.63 \\
$5-3$ & 23.1 & 23.5 & 22.7 & 1.87 \\
$5-4$ & 148.7 & 153.7 & 140.3 & 6.01 \\
$6-4$ & 35.9 & 36.3 & 34.5 & 3.96 \\
$6-5$ & 155.6 & 161.3 & 145.7 & 6.79 \\
$7-5$ & 51.8 & 52.3 & 49.3 & 4.99 \\
\hline
\end{tabular}

Notes. ${ }^{(a)}$ Using the $\mathrm{P}(1)$ transition. ${ }^{\left({ }^{b}\right)}$ Coppola et al. (2011). ${ }^{(c)}$ Table V in Partridge \& Langhoff (1981). ${ }^{(d)}$ Percent difference between current results and Partridge \& Langhoff (1981).

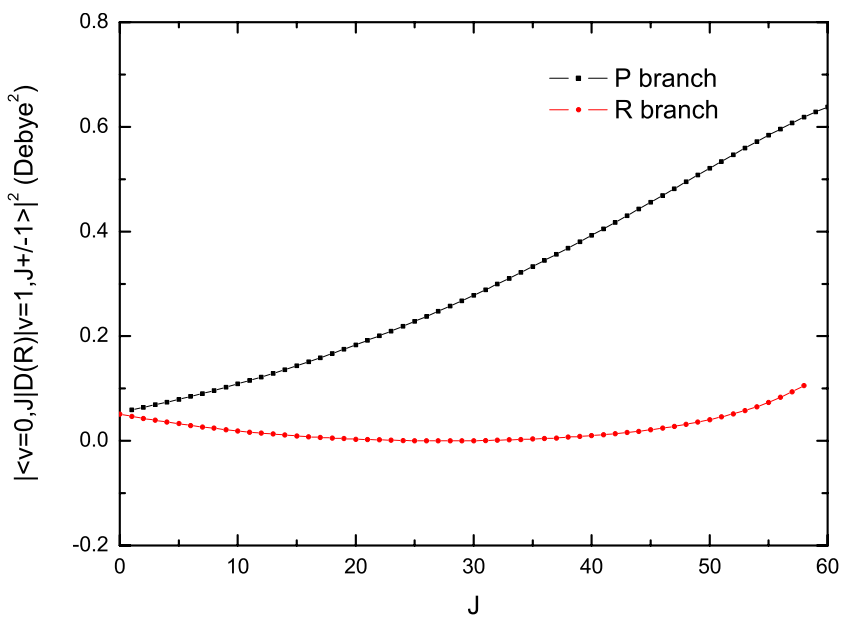

Fig. 2. Dipole moment matrix elements for the P-branch and R-branch rovibrational transitions for ${ }^{7} \mathrm{LiH}$ for the $v=0$ to $v=1$ transition.

one to two orders of magnitude smaller. According to the cool dwarf atmosphere models of Lodders (1999), the abundances of $\mathrm{LiH}$ and $\mathrm{LiCl}$, as well as $\mathrm{LiOH}$, are comparable at $1800 \mathrm{~K}$, but for higher temperatures, $\mathrm{LiH}$ becomes the dominant Li-bearing molecule. Weck et al. (2004) suggest that it would be difficult to detect $\mathrm{LiCl}$ in cool dwarfs due to strong absorption features from other more abundant molecules (e.g., water) in the same wavelength range. $\mathrm{LiH}$, with its somewhat higher opacity,might be a better candidate for detecting lithium molecules.

\section{Conclusions}

The rovibrational energy levels of ${ }^{7} \mathrm{LiH}$ were computed with an empirical potential generated from a direct fit of available spectroscopic line positions. The computed vibrational energies and transition energies are found to be in very good agreement with experiments. Oscillator strengths and transition probabilities were also computed and used to construct a complete rovibrational line list. The transition energies were evaluated against available experimental data and found to be reliable to better than $0.03 \mathrm{~cm}^{-1}$. The line list was used to compute an LTE radiative cooling function for $\mathrm{LiH}$ and to explore its infrared opacity. Among all dominant Li-bearing molecules, $\mathrm{LiH}$ is a better candidate for detecting lithium in cool dwarf atmospheres.

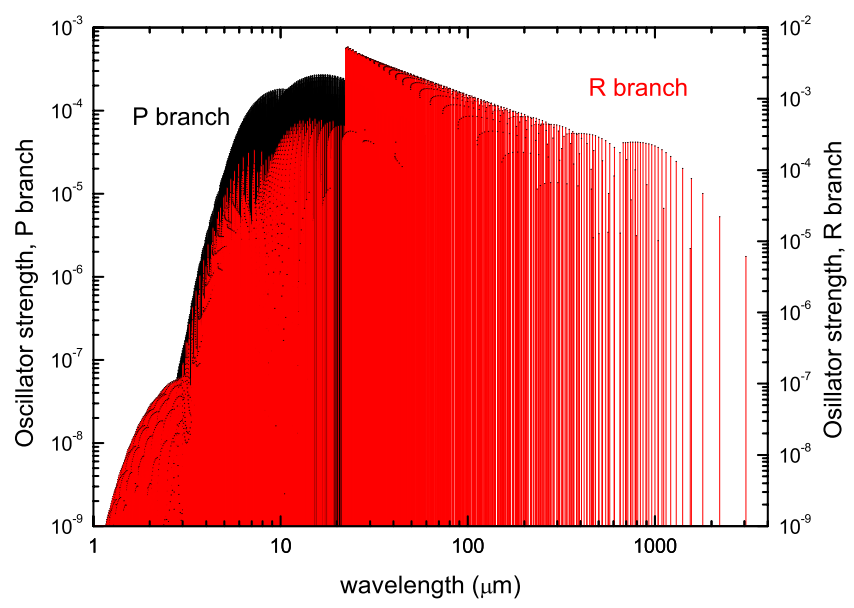

Fig. 3. Oscillator strengths for the $X^{1} \Sigma^{+} \leftarrow X^{1} \Sigma^{+}$transition of ${ }^{7} \mathrm{LiH}$ versus wavelength. R-branch (red) and P-branch (black).

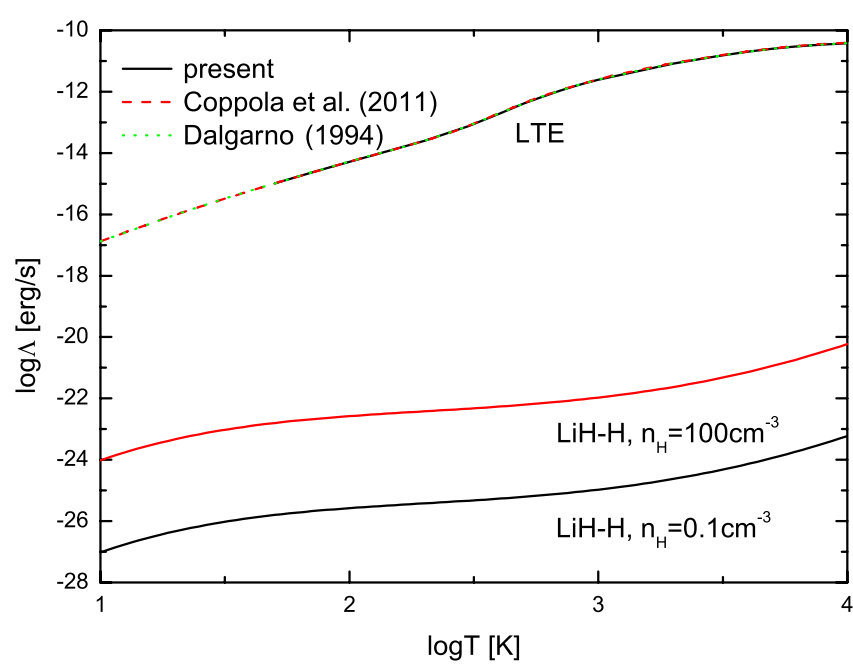

Fig. 4. Radiative cooling functions of $\mathrm{LiH}$ in the LTE (upper three lines) and the low-density limit $\left(n_{\mathrm{H}} \leq 100 \mathrm{~cm}^{-3}\right.$, lower solid red and black lines) considering only $\mathrm{H}$ collisions.

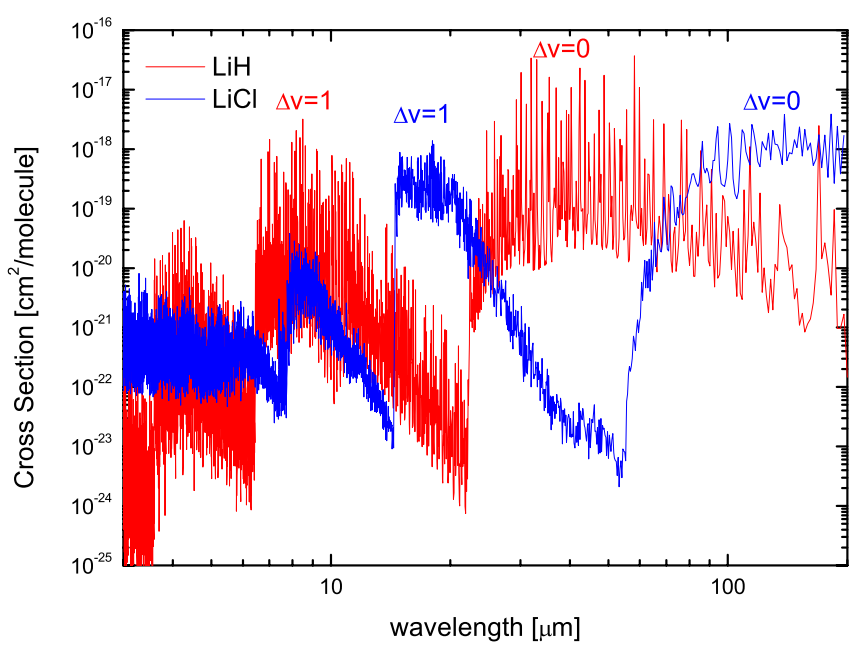

Fig. 5. Opacity of the $\mathrm{LiH}$ (red) rovibrational transitions in the infrared compared to the $\mathrm{LiCl}$ (blue) opacity for a temperature of $1800 \mathrm{~K}$ and a pressure of 100 atms. 
Acknowledgements. The work of P.C.S. was partially supported by NSF grant AST-0607733 and NASA grant NNX07AP12G. Y.B.S. acknowledges travel support by the International Cooperation and Exchange Foundation of CAEP. We thank the referee for helpful comments that improved the manuscript.

\section{Appendix: Collisional excitation of LiH}

While in this work, we present an LTE radiative cooling function (i.e., valid within the high-density limit), Bougleux \& Galli (1997) have approximated a density-dependent non-LTE (NLTE) cooling function (see also Galli \& Palla 1998). In this approach, the NLTE cooling function is parameterized in terms of the LTE radiative cooling function and a collisional cooling function in the zero-density limit. For applications to the early Universe, they considered only collisions due to atomic hydrogen. Unfortunately, rotational or vibrational excitation rate coefficients of $\mathrm{LiH}$ due to $\mathrm{H}$ are not available, requiring Bougleux \& Galli (1997) to adopt the LiH-He calculations of Jendrek \& Alexander (1980), but adjusted to emulate LiH-H collisions via mass-scaling of the collisional rate coefficients. The massscaling approach is typically adopted when rate coefficients due to $\mathrm{He}$ impact are used to approximate para- $\mathrm{H}_{2}$ impact. Recent work on other molecules suggests this approach is questionable at best (e.g., Cernicharo et al. 2011) even though the PESs for $\mathrm{He}$ - and $\mathrm{H}_{2}$-complexes are expected to be similar, being characterized by weakly bound van der Waals minima. Collision complexes involving $\mathrm{H}$, on the other hand, typically involve very deep wells leading to very fast exothermic reactive channels. As a consequence, mass-scaling to deduce $\mathrm{H}$-impact excitation rate coefficients from $\mathrm{He}$ - or $\mathrm{H}_{2}$-impact data is likely to be a poor approximation.

For general astrophysical applications, collisions due to the most abundant species, $\mathrm{H}, \mathrm{H}_{2}, \mathrm{He}, \mathrm{e}^{-}$, and $\mathrm{H}^{+}$, should be included in an NLTE cooling function, though $\mathrm{H}_{2}$ dominates in most interstellar environments. LiH-He collisional excitation calculations have been more recently given by Taylor \& Hinde (1999, 2005) and Bodo et al. (2001a), and discussed in a review of lithium chemistry by Bodo et al. (2003). The reactive process

$$
\mathrm{LiH}+\mathrm{H} \rightarrow \mathrm{H}_{2}+\mathrm{Li}
$$

and the H-exchange process

$$
\mathrm{LiH}_{\mathrm{a}}+\mathrm{H}_{\mathrm{b}} \rightarrow \mathrm{LiH}_{\mathrm{b}}+\mathrm{H}_{\mathrm{a}}
$$

have been considered in a number of recent studies (Prudente et al. 2009; Bovino et al. 2009), but to date, inelastic collisional excitation of $\mathrm{LiH}$ due to $\mathrm{H}$ has not been investigated because it is a very computationally demanding problem that would require simultaneous treatment with reactions (13) and (14). We are unaware of any inelastic data due to $\mathrm{H}_{2}, \mathrm{e}^{-}$, or $\mathrm{H}^{+}$impacts, though an excited state PES for the $\mathrm{LiH}_{2}^{+}$complex is available (Bodo et al. 2001b), which could be used later. It is therefore, not currently possible to construct an accurate, comprehensive NLTE cooling function for LiH. Figure 4 gives an indication of the expected range of the NLTE cooling function.

\section{References}

Bernath, P. F. 1995, Spectra of atoms and molecules (Oxford University Press) Bodo, E., Gianturco, F. A., \& Martinazzo, R. 2001a, Chem. Phys., 271, 309 Bodo, E., Gianturco, F. A., \& Martinazzo, R. 2001b, J. Phys. Chem. A, 105, 10986

Bodo, E., Gianturco, F. A., \& Martinazzo, R. 2003, Phys. Rep., 384, 85

Bottcher, C., \& Dalgarno, A. 1974, Proc. Roy. Soc. London, Ser. A, 340, 187

Bougleux, E., \& Galli, D. 1997, MNRAS, 288, 638

Bovino, S., Wernli, M., \& Gianturco, F. A. 2009, ApJ, 699, 383

Bovino, S., Tacconi, M., Gianturco, F. A., Galli, D., \& Palla, F. 2011, ApJ, 731, 107

Cernicharo, J., Spielfiedel, A., Balança, C., et al. 2011, A\&A, 531, A103

Chan, Y. C., Harding, D. R., \& Stwalley, W. C. 1986, J. Chem. Phys., 85, 2436

Coppola, C. M., Lodi, L., \& Tennyson, J. 2011, MNRAS, 415, 487

Coxon, J. A., \& Dickinson, C. S. 2004, J. Chem. Phys., 121, 9378

Dalgarno, A. 1994, unpublished

Dulick, M., Zhang, K. Q., Guo, B., \& Bernath, P. F. 1998, J. Mol. Spectrosc., 188,14

Dulick, M., Bauschlicher, C. W. Jr., Burrows, A., et al. 2003, ApJ, 594, 651

Friedel, D. N., Kemball, A., \& Fields, B. D. 2011, ApJ, 738, 37

Galli, D., \& Palla, F. 1998, A\&A, 335, 403

Gianturco, F. A., Giorgi, P. G., Berriche, H., \& Gadea, F. X. 1996, A\&AS, 117, 377

Herzberg, G. 1989, Molecular Structure I, Spectra of Diatomic Molecules, 2nd edn. (Krieger Pub Co.)

Holka, F., Szalay, P. G., Fremont, J., et al. 2011, J. Chem. Phys., 134, 094306

Jendrek, E. F., \& Alexander, M. H. 1980, J. Chem. Phys., 72, 6452

Johnson, B. R. 1977, J. Chem. Phys., 67, 4086

Lepp, S., Stancil, P. C., \& Dalgarno, A. 2002, J. Phys. B: At. Mol. Opt. Phys., 35, R57

Lodders, K. 1999, ApJ, 519, 793

Partridge, H., \& Langhoff, S. R. 1981, J. Chem. Phys., 74, 2361

Prudente, F. V., Marques, J. M. C., \& Maniero, A. M. 2009, Chem. Phys. Lett., 474, 18

Schöier, F. L., van der Tak, F. F. S., van Dishoeck, E. F., \& Black, J. H. 2005, A\&A, 432, 369

Stancil, P. C., Lepp, S., \& Dalgarno, A. 1996, ApJ, 458, 401

Stwalley, W. C., \& Zemke, W. T. 1993, J. Phys. Chem. Ref. Data, 22, 87

Taylor, B. K., \& Hinde, R. J. 1999, J. Chem. Phys., 111, 973

Taylor, B. K., \& Hinde, R. J. 2005, J. Chem. Phys., 122, 074308

Tung, W.-C., Pavanello, M., \& Adamowicz, L. 2011, J. Chem. Phys., 134, 064117

Weck, P. F., Schweitzer, A., Kirby, K., Haushildt, P. H., \& Stancil, P. C. 2004, ApJ, 613, 567

Weissbluth, M. 1978, Atoms and Molecules (Academic press)

Yan, Z. C., Babb, J. F., Dalgarno, A., \& Drake, G. W. F. 1996, Phys. Rev. A, 54, 2824 